\title{
High Infectivity of Salmonella typhimurium newly infected by the coll factor
}

\author{
By B. A. D. STOCKER, SYLVIA M. SMITH AND H. OZEKI \\ Guinness-Lister Research Unit, Lister Institute of Preventive Medicine, \\ Chelsea Bridge Road, London S.W. I
}

(Received 13 April 1962)

\begin{abstract}
SUMMARY
In $18 \mathrm{hr}$. incubation of broth inoculated with a majority of bacteria of Salmonella typhimurium LT2 col-, i.e. non-colicinogenic, and a minority of strain LT2 (colI), i.e. carrying colicine factor colI, 30-70\% of the colbacteria acquired colI; this increased to 50-90\% after $2 \mathrm{hr}$. of secondary incubation after tenfold dilution with broth. These cultures, containing a high proportion of bacteria newly infected by colI, transmitted colI to about $50 \%$ of the bacteria of a $\mathrm{col}^{-}$strain in $1 \mathrm{hr}$. (whereas pure cultures of LT2 (colI) transmitted to only $0.01 \%$ ); they are termed HFC (high-frequency colicinogeny-transferring). An inoculum containing 1-B recently infected bacteria from an HFC preparation of a streptomycin-sensitive strain sufficed to transmit colI to a resistant strain in streptomycin broth. We infer that 30-100\% of bacteria newly infected by colI are 'competent donors', able to transmit colI. By the same test the proportion of competent donors in LT2 (colI) strains was only $0.02 \%$. Inoculum size, ratio of inoculum components, motility, aeration and secondary incubation affected the HFC property of mixed cultures in a way explicable by the need for the epidemic spread of $\mathrm{colI}$ in the $\mathrm{col}^{-}$component to reach a peak at the time of testing. The rate of loss of the HFC property on further growth indicated that for 3-7 generations the progeny of newly infected bacteria are competent donors. Transmission was associated with clumping and pairing. Bacteria of an HFC preparation pair with $10 \%$ of an acceptor population within $2 \mathrm{~min}$. of mixing; completion of transfer requires $2 \frac{1}{2}-30 \mathrm{~min}$. Non-availability of nutrients and some growth inhibitors interfere with transfer.

We conclude that colI multiplies autonomously in newly infected bacteria and their immediate progeny and enables them to conjugate, but does not confer ability to conjugate in established colicinogenic strains; perhaps it is then integrated into the host chromosome.
\end{abstract}

\section{INTRODUCTION}

In a previous paper (Ozeki, Stocker \& Smith, 1962) we showed that the genetic factors or episomes colI, colE2, etc., which determine, respectively, the production of colicines I, E2, etc., could be transferred from standard colicinogenic strains of Escherichia coli or Shigella sonnei to Salmonella typhimurium strain LT2. When a broth culture of $S$. typhimurium LT2 (colI)-i.e. an LT2 line producing colicine Ias donor strain was incubated with a broth culture of a non-colicinogenic (acceptor) LT2 strain, $<0.1 \%$ of the acceptor bacteria became colicinogenic during incubation for $1 \mathrm{hr}$., but after incubation for $18 \mathrm{hr}$. about $50 \%$ of them had done so. We 
showed that in a broth culture of LT2 (colI) only about 1 bacterium in 5000 was a 'competent donor', able to transmit the coll factor by contact to a non-colicinogenic acceptor bacterium. This accounted for the low proportion of acceptor bacteria which acquired colicinogeny in $\mathbf{l} \mathrm{hr}$. contact with a donor strain. We attributed the high proportion of acceptor bacteria which acquired colI on long incubation of a mixture, to serial transmission of coll amongst the acceptor bacteria, initiated by the few acceptor bacteria which acquired the factor during the first hour. We explained this 'epidemic spread' by the hypothesis that many or perhaps all bacteria which newly acquire coll become competent donors, able to pair with, and transmit colI to, acceptor bacteria.

We were led to this hypothesis by the observation that overnight broth cultures inoculated directly from one particular Dorset egg stock culture of a donor strain, CL114, transmitted colI to a considerable proportion (about $10 \%$ ) of acceptor bacteria in $1 \mathrm{hr}$. When material from this stock slope was streaked out only about $\mathbf{5} \%$ of the Salmonella paratyphi B colonies obtained were colicinogenic; broth cultures inoculated from such colicinogenic colonies transmitted colicinogeny no better than did broth cultures of LT2 $(\mathrm{colI})$. But broth cultures inoculated with a mixture of the purified colicinogenic and non-colicinogenic components of strain CL114, in the same ratio as was found in the stock slope, transmitted to about $10 \%$ of an acceptor culture in $1 \mathrm{hr}$. These broth cultures inoculated with mixtures of colicinogenic and non-colicinogenic bacteria, in the ratio of $1: 20$, when streaked out after overnight incubation yield about $\mathbf{5 0} \%$ of colicinogenic colonies, as a result of the acquisition of colI by bacteria of the originally non-colicinogenic component inoculated. We therefore surmised that such newly infected bacteria might be much more effective as donors of colicinogeny than bacteria which had carried colI for many generations.

The experiments to be described show that in strain LT2 a high proportion (half or more) of bacteria which have just acquired coll become competent donors, and that this competence persists in their progeny for between 2 and 7 generations. We also describe the kinetics of transfer of colI, show that it is associated with the formation of pairs and clumps and describe the effect of various environmental factors and metabolic inhibitors on the process of transfer.

In all experiments, except the initial ones on the Salmonella paratyphi B strain mentioned above, we used $S$. typhimurium strain LT2 carrying the colI factor derived from Shigella sonnei strain P9 (Ozeki et al. 1962). In some experiments the strain inoculated into broth with LT2 (colI) was not non-colicinogenic but, instead, LT2 (colE2); however, the presence of colE2 in these bacteria seemed to have no effect on the behaviour of colI in them. In a later paper (Smith, Ozeki \& Stocker, in preparation) we shall describe the transmission of colE2, etc., by such colicinogenic bacteria newly infected by colI.

\section{METHODS}

In general the strains, media, tests for colicinogeny and other methods used were as described by Ozeki et al. (1962). Some additional strains used are listed in Table 1. The major component of a Dorset egg stock culture labelled CL114 was a Salmonella paratyphi $\mathrm{B}$ strain, of unknown origin because the stock culture concerned purported to be a colicinogenic Escherichia coli strain; colicinogenic sublines of this 
S. paratyphi B strain (producing colicine I) we designate CL114 $\left(\right.$ colI $\left._{2}\right)$, non-colicinogenic sublines CL114 col-. Except where the contrary is indicated the colicine I factor used was that derived from Shigella sonnei P9 (Ozeki et al. 1962). The colicine I factor of Salmonella paratyphi B, strain CL114 is indicated colI ${ }_{2}$. The colicines determined by these two factors are indistinguishable.

Table 1. Bacterial strains used*

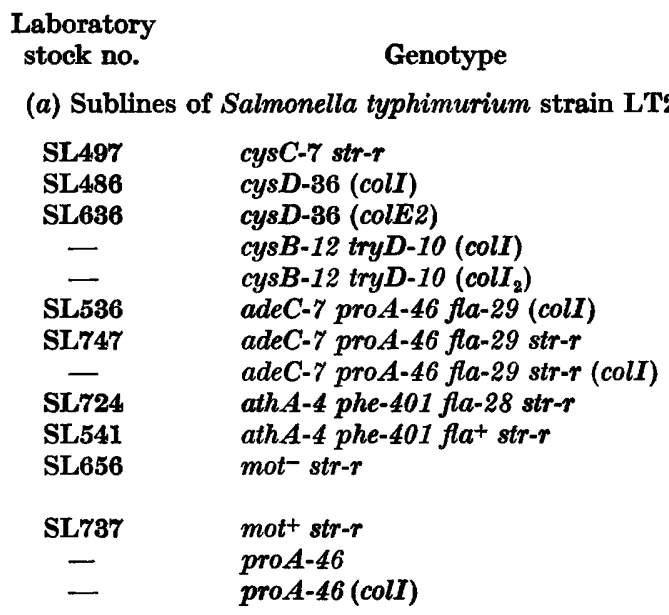

Remarks

(b) Sublines of $S$. typhimurium var. copenhagen $O=$ SW541 (Stocker et al. 1958)

$\begin{array}{ll}\text { SL178 } & f l a^{-} \text {str-r } \\ \text { SL722 } & f l a^{-} \text {str-r cured of an A phage } \\ \text { SL723 } & f l a^{+} \text {str-r }\end{array}$

\section{Non-lysogenic $\overline{\mathrm{fa}^{+}}$transductant of SL722}

Requirements for growth: ade = adenine; ath = adenine and thiamine; $c y s=$ cystine; $p h e=$ phenylalanine; pro $=$ proline; $t$ ry $=$ tryptophan.

str-r $=$ streptomycin-resistant (1 mg./ml.).

$f l a^{-}$or $f l a-28$, etc. $=$ non-flagellated, non-motile; $f a^{+}=$flagellated.

mot $=$ flagellated, non-motile ('paralysed'); mot $^{+}=$not paralysed.

(colI) = carrying coll factor derived from Shig. sonnei P9 (Ozeki et al. 1962).

$\left(\operatorname{colI}_{2}\right)=$ carrying coll factor derived from $S$. paratyphi B, CL114.

(colE2) = carrying colE2 factor derived from Shig. sonnei $\mathbf{P 9}$.

Most of the auxotrophic mutants used were obtained from the Department of Genetics, Carnegie Institution of Washington at Cold Spring Harbor (see Demerec et al. 1956; Clowes, 1958; Miyake \& Demerec, 1960). Non-flagellated, streptomycin-resistant and colicinogenic stocks were then derived by us.

* See also the strains listed in Table 1 of Ozeki et al. (1962).

In experiments in which motile and non-motile variants of Salmonella typhimurium strains were compared (Table 3) we either used non-motile strains originally isolated as such, and motile sublines obtained from them by mutation or transduction (Stocker, Zinder \& Lederberg, 1953); or selected non-motile variants (either non-flagellated or 'paralysed') from motile strains by exposure to phage $\phi \chi$ (Sertic \& Boulgakov, 1936; Meynell, 1961). In experiments in which we used non-motility as a phenotypic or genotypic marker, we chose non-motile strains in which motile bacteria arising by phenotypic variation (Quadling \& Stocker, 1957) were absent or rare. The semi-solid medium used was that of Stocker et al. (1953) with a decreased 
agar concentration: i.e. peptone, 10 g.; Difco gelatine, 80 g.; Difco agar, 3 g.; sodium chloride, 5 g.; water, $1000 \mathrm{ml}$.

Bacteria visibly 'labelled' with formazan granules (Hartman, Mudd, Hillier \& Beutner 1953) were obtained by growth in broth containing 2,3,5-triphenyltetrazolium chloride, $0.01 \%(\mathrm{w} / \mathrm{v})$; after $24 \mathrm{hr}$. at $37^{\circ}$ the culture was diluted tenfold into broth containing twice this concentration of the tetrazolium compound. After a further $2 \mathrm{hr}$. incubation period at least $50 \%$ of the bacteria contained visible granules.

High-speed mixing of broth cultures was effected in an M.S.E. blendor as described by Stocker \& Campbell (1959). Treatment for 2 min. at full speed (about 12,000 rev./ min.) sufficed to break up all clumps.

To see whether Salmonella typhimurium newly infected by the colI factor developed any new antigen (compare the $\mathrm{f}^{+}$antigen of Escherichia coli described by Ørskov \& Ørskov, 1960) a rabbit was given two courses $(0.5 \mathrm{ml}$., then $1 \mathrm{ml}$. weekly for 3 weeks; later five $1.0 \mathrm{ml}$. inocula during $2 \frac{1}{2}$ months) of intravenous inoculations of a formalinized broth culture of an HFC preparation (see below) of strain LT2 pro $A-46$. O-agglutinable suspensions were made by deflagellation (by high-speed mixing) and washing of a broth culture of strain proA-46, and of an HFC culture of the same strain.

\section{RESULTS}

When material from a Dorset egg stock culture labelled CL114 was plated on nutrient agar, only about $5 \%$ of the Salmonella paratyphi $\mathbf{B}$ colonies obtained were colicinogenic, producing colicine I; but when broth inoculated from the same slope was streaked out after overnight incubation more than half the $S$. paratyphi B colonies obtained were colicinogenic (Table 2, Expt. 1). A similar increase in the proportion of colicinogenic bacteria occurred during overnight incubation of broth inoculated with an artificial mixture, in the ratio $1: 20$, of the colicinogenic and the non-colicinogenic $S$. paratyphi B components of stock culture CL114 (Table 2, Expt. 4); or when the inoculum was a similar mixture of genetically labelled LT2 sub-lines, one non-colicinogenic and the other carrying colI, derived either from $S$. paratyphi B, CL114 (Table 2, Expt. 7) or from Shigella sonnei P9 (Table 2, Expt. 6). In the latter two experiments testing of the nutritional character of the colonies showed that the increase in the proportion of colicinogenic organisms from 5 to $50-80 \%$ resulted from the acquisition of the colI factor by bacteria of the originally non-colicinogenic strain, and not from overgrowth by the donor bacteria.

Donor preparations, i.e. broth cultures grown overnight from mixed inocula of colicinogenic and non-colicinogenic bacteria in the ratio $1: 20$, and cultures inoculated only with the colicinogenic component, were compared in respect of their ability to transmit the coll factor to a streptomycin-resistant acceptor strain during $1 \mathrm{hr}$. contact (Table 2). The pure cultures of colicinogenic strains transmitted to less than $\mathbf{0 . 2} \%$ of the acceptor bacteria; whereas donor preparations grown from mixed inocula infected 10-21\%. The same result was obtained whether the Salmonella strain used for the donor preparations was Salmonella paratyphi B, CL114 or $S$. typhimurium LT2; and whether the colI agent was that derived from CL114 or that from Shigella sonnei P9. As the behaviour in strain LT2 of the colI agent from P9 has been fully investigated (Ozeki, Stocker \& Smith, 1962) we used this system in all later experiments. In experiments on the transmission of colI by donor prepara- 
tions, bacteria of the initially non-colicinogenic component of the donor mixture accepted colI during the growth of the donor mixture; when the donor preparation was tested for its ability to transmit colI to a third strain (usually marked by streptomycin-resistance) these newly infected bacteria now acted as donors. We shall therefore call the non-colicinogenic strain used as one component of a donor mixture the intermediate strain, since it first accepts colI, then transmits it.

\section{Table 2. Behaviour of cultures inoculated with colicinogenic or non-colicinogenic strains, and with mixtures thereof}

Broths inoculated either directly from stock culture slopes, or with about $5 \times 10^{4}$ and $10^{6}$ bacteria/ml. of the indicated strains, were streaked on nutrient agar before and after $18 \mathrm{hr}$. incubation, and the proportion of colicinogenic colonies determined. To test their transmitting ability the incubated broth cultures were mixed in equal parts with a culture of a streptomycin-resistant, non-colicinogenic acceptor strain (SL178); after $1 \mathrm{hr}$. incubation the mixture was streaked on steptomycin agar and the resulting acceptor colonies were tested for colicinogeny.

\begin{tabular}{|c|c|c|c|c|}
\hline \multirow[b]{2}{*}{$\begin{array}{l}\text { Expt. } \\
\text { no. }\end{array}$} & \multirow[b]{2}{*}{ Inoculum } & \multicolumn{2}{|c|}{ Colicinogenic bacteria ( \%) } & \multirow{2}{*}{$\begin{array}{c}\% \text { str-r } \\
\text { acceptors } \\
\text { acquiring } \\
\text { coll in } 1 \mathrm{hr} \text {. }\end{array}$} \\
\hline & & $\begin{array}{c}\text { Before } \\
\text { incubation }\end{array}$ & $\begin{array}{l}\text { After } 18 \mathrm{hr} . \\
\text { incubation }\end{array}$ & \\
\hline 1 & CL114 'stock'* & About 5 & 68 & 12 \\
\hline 2 & $\operatorname{CL114}\left(\operatorname{colI}_{2}\right) \dagger$ & 100 & 100 & $\mathbf{0} \cdot \mathbf{1}$ \\
\hline $\mathbf{3}$ & CL114 col- $_{\ddagger}^{-}$ & $\mathbf{0}$ & $\mathbf{0}$ & $\mathbf{0}$ \\
\hline 4 & $\begin{array}{l}\text { CL114 }\left(\mathrm{colI}_{2}\right) \text { and } \mathrm{CLI14}^{-\mathrm{col}^{-}} \text {, } \\
\text { in ratio } 1: 20\end{array}$ & 5 & 60 & 16 \\
\hline 5 & LT2 cys try (colI) & 100 & 100 & $\mathbf{0} \cdot \mathbf{1}$ \\
\hline 6 & $\begin{array}{l}\text { LT2 cys try (colI) and LT2 wild- } \\
\text { type col-, in ratio } 1: 20\end{array}$ & 5 & 78 & 11 \\
\hline 7 & $\begin{array}{l}\text { LT2 cys try }\left(\operatorname{colI}_{2}\right) \text { and LT2 wild- } \\
\text { type } \operatorname{col}^{-} \text {, in ratio } 1: 20\end{array}$ & $\mathbf{5}$ & $\mathbf{5 4}$ & 21 \\
\hline
\end{tabular}

The preponderance of non-colicinogenic Salmonella paratyphi $\mathrm{B}$ in the stock culture slope CL114 was found to result from an instability of the colicinogenic state in this strain, since Dorset egg slopes inoculated with CL114 ( $\left.\operatorname{colI}_{2}\right)$, i.e. the purified colicinogenic component of the original culture, after several weeks at room temperature yielded a majority of non-colicinogenic colonies, as also did clones of CL114 col- re-infected with $\mathrm{colI}_{2}$, or infected with the colI factor from Shigella sonnei P9. A clone of CL114 (colI) (colE2) after some weeks gave mainly CL114 col $^{-}$and a few CL114 (colI) and CL114 (colI) (colE2). The instability of colicinogeny for colI and colE2 in Salmonella paratyphi B, CL114 contrasts with their stability in S. typhimurium LT2.

Incubation of acceptor bacteria for up to 2 days with supernatant fluids from 'donor preparations' (i.e. overnight mixed cultures of colicinogenic and noncolicinogenic strains) sterilized by filtration or chloroform treatment, did not result in any detectable acquisition of colicinogeny; and the bacteria from donor preparations spun down and resuspended in fresh broth transmitted colicinogeny as well as did the original preparations. This is what would be expected if, as we believe, transmission of colicinogeny occurs only by cell conjugation. 


\section{Factors affecting the transmitting ability of donor preparations}

As we surmised that the high transmitting ability of overnight mixed cultures resulted from the presence of a high proportion of bacteria of the intermediate strain which had very recently acquired the coll factor, we sought to obtain donor preparations in which a high proportion of the bacteria of the intermediate strain were colicinogenic; in general such donor preparations transmitted colicinogeny well, i.e. infected a high proportion of the bacteria of the acceptor strain during $1 \mathrm{hr}$. contact. A method which generally gave satisfactory results was to inoculate $10 \mathrm{ml}$. of broth with about $5 \times 10^{5}$ donor bacteria and about $10^{7}$ intermediate bacteria, and to incubate at $37^{\circ}$ without aeration by shaking for $18 \mathrm{hr}$., by which time the proportion of colicinogenic bacteria had increased from $5 \%$ to $30-70 \%$. When this preparation was diluted tenfold in broth and incubated for a further $2 \mathrm{hr}$., the proportion of colicinogenic bacteria further increased to 50-90\%, presumably as a result of the rapid transmission of the coll factor by the recently infected bacteria of the intermediate strain. The donor preparations so obtained transmitted colicinogeny to about $20 \%$ of the bacteria of an acceptor culture during $15 \mathrm{~min}$. contact, and to $50 \%$ in $60 \mathrm{~min}$.; we shall term them HFC (high frequency colicinogeny-transferring) preparations.

Composition of inoculum of donor preparation. To determine the effect of varying the composition of the inoculum, the $10 \mathrm{ml}$. tube of broth used for growth of a donor preparation received the standard inoculum of intermediate bacteria, i.e. $10^{6}$ bacteria/ml., and graded numbers of donor bacteria. When the inoculum of donor bacteria was $<5000 / \mathrm{ml}$. (and the donor:intermediate ratio was $<1: 200$ ) $<1 \%$ of the intermediate bacteria became colicinogenic after $18 \mathrm{hr}$. and the transmitting ability of the preparation was very low; with inocula of donor bacteria between 5000 and $10^{6} / \mathrm{ml}$. (and donor:intermediate ratios between 1:200 and 1:1) a high proportion of the intermediate bacteria became colicinogenic during overnight incubation, and ability to transmit was high; with a donor:intermediate ratio of 10:1 or $100: 1$, the proportion of intermediate bacteria which acquired colicinogeny after overnight incubation was low (about $3 \%$ ). We therefore adopted a donor : intermediate ratio of $1: 20$ as the inoculum for routine growth of HFC preparations. Tubes inoculated with $10^{6}, 10^{7}, 10^{8}$ or $10^{9}$ bacteria/ml. with the donor:intermediate ratio constant at 1:20, gave HFC preparations containing 39-68\% colicinogenic bacteria, and transmitting colI to $12-24 \%$ of the acceptor bacteria in $30 \mathrm{~min}$.

Effect of aeration by shaking. When broth inoculated with a mixture of colicinogenic and non-colicinogenic bacteria was shaken during the overnight incubation, transfer of colicinogeny to the non-colicinogenic bacteria was greatly decreased, as compared with an unshaken control (Ozeki et al. 1962); such shaken cultures, as might be expected, transferred colicinogeny very poorly during $60 \mathrm{~min}$. contact with an acceptor. By contrast, when a donor preparation, grown by overnight incubation without shaking from a standard inoculum, was shaken during the $2 \mathrm{hr}$. final incubation stage, there was a further increase in the proportion of colicinogenic intermediate bacteria, e.g. from 63 to $88 \%$, as compared with an increase to $94 \%$ in an unshaken control; and the HFC preparation so obtained transmitted well.

Effect of medium. HFC preparations could be prepared by incubating donor and intermediate bacteria in a defined glucose + ammonia + salts medium supplemented 
with any required growth factors; but the percentage of intermediate bacteria which acquired colicinogeny was more variable than when broth was used. In the defined medium, though not in broth, transfer of colicinogeny to the intermediate bacteria occurred even when the mixture was shaken during the overnight incubation. Such cultures transmitted colicinogeny at high frequency.

Effect of bacterial motility. When the non-colicinogenic (intermediate) component of the mixed inoculum was a non-motile strain, either non-flagellated or 'paralysed' (Stocker et al. 1953), the proportion of the intermediate bacteria which acquired colicinogeny during the primary overnight and the secondary $2 \mathrm{hr}$. incubation was greatly decreased as compared with a control mixture in which the intermediate was a motile form of the same strain; e.g. $<1 \%$ colicinogenic after the $2 \mathrm{hr}$. incubation as compared with about $70 \%$ in the control (Table 3). However, when secondary incubation was continued for $24 \mathrm{hr}$. the proportion of colicinogenic intermediate bacteria increased, e.g. to $50 \%$. When the intermediate component was motile, satisfactory HFC preparations were obtained even when the donor component was non-motile.

Table 3. Effect of motility or non-motility of intermediate strain on proportion of intermediate bacteria acquiring coll

Proportion of intermediate bacteria colicinogenic determined on samples plated on streptomycin nutrient agar after $18 \mathrm{hr}$. primary incubation, and after 2 and $24 \mathrm{hr}$. secondary incubation subsequent to tenfold dilution in broth.

Donor: LT2 ade $C-7$ pro $A-46$ fla- (colr).

Intermediate: as indicated, in all cases col $^{-}$and $s t r-r$.

Inoculum: $5 \times 10^{4}$ donors $/ \mathrm{ml}$., $10^{6}$ intermediates $/ \mathrm{ml}$.

\begin{tabular}{|c|c|c|c|}
\hline & \multicolumn{3}{|c|}{$\begin{array}{l}\text { Percentage in intermediate bacteria } \\
\text { colicinogenic after: }\end{array}$} \\
\hline Intermediate strain & $\begin{array}{c}18 \mathrm{hr} . \\
\text { primary } \\
\text { incubation }\end{array}$ & $\begin{array}{c}\mathbf{2} \mathrm{hr} . \\
\text { secondary } \\
\text { incubation }\end{array}$ & $\begin{array}{c}24 \mathrm{hr} \text {. } \\
\text { secondary } \\
\text { incubation }\end{array}$ \\
\hline & $<0.5$ & $<1$ & 13 \\
\hline transductant of SL722) & $\mathbf{8}$ & 16 & 18 \\
\hline & $<0.5$ & $<0.5$ & 50 \\
\hline mutant of SL656) & 40 & 70 & 80 \\
\hline$)^{*}$ & 11 & 13 & 15 \\
\hline mutant of SL724) & $\mathbf{8 4}$ & 83 & 91 \\
\hline
\end{tabular}

* A very few motile bacteria were seen on microscopy of this culture.

Effect of secondary incubation. In the standard procedure for making an HFC preparation an overnight mixed culture was diluted tenfold in broth and incubated for $2 \mathrm{hr}$. This secondary incubation resulted in an increase in the proportion of colicinogenic bacteria and in a greater increase in transmitting ability. For instance, in one experiment the proportion of colicinogenic intermediate bacteria increased from 27 to $86 \%$ during secondary incubation; samples taken before and after secondary incubation (and adjusted to the same bacterial count, after blendor treatment to break up clumps) infected 5 and $40 \%$, respectively, of the bacteria of a streptomycin-resistant acceptor strain in $1 \mathrm{hr}$. contact. No large increase in the 
proportion of colicinogenic intermediate bacteria occurred when the overnight mixture was incubated for a further $2 \mathrm{hr}$. without addition of fresh broth. When the overnight mixed culture was diluted $1 / 100$ or 1/1000 in fresh broth, the increase in proportion of colicinogenic bacteria during incubation for $2 \mathrm{hr}$. was less than when the culture was diluted $1 / 10$ as in the standard procedure.

\section{Cell pairs and clumps associated with transfer of the colI factor}

Microscopy of HFC preparations showed many clumps; e.g. about $60 \%$ of the bacteria in clumps of 2 to 20 , average about 4, bacteria. No clumps were seen in broth cultures of non-colicinogenic or of pure colicinogenic strains, nor in mixtures of such cultures incubated for $1 \mathrm{hr}$. Shaking by hand did not break up the clumps; but after $2 \mathrm{~min}$. treatment in a high-speed blendor less than $0.1 \%$ of the bacteria were in clumps. After $\mathbf{3 0}$ min. incubation of the blendor-treated HFC suspension, $14 \%$ of the bacteria were once more in clumps, on an average of three bacteria. To prove that the motile bacteria of an HFC preparation adhered to bacteria of an acceptor strain we mixed such an HFC preparation with a non-motile acceptor grown in tetrazolium broth, so that most bacteria contained a visible formazan granule or granules. Active movement of pairs of bacteria, one containing a formazan granule, proved that donor bacteria became attached to acceptor bacteria. Granule-bearing acceptor bacteria could also be seen attached to the clumps characteristic of $\mathrm{HFC}$ preparations. The use of this technique also showed that the bacteria of an HFC preparation adhered about as readily to bacteria of a colicinogenic strain, already carrying colI as they did to bacteria of the ordinary noncolicinogenic acceptor strain.

We also used an indirect test to detect the adherence of motile bacteria of an HFC preparation to acceptor bacteria. The acceptor strain used (SL 747) was streptomycin-resistant and completely non-motile. After incubation of the HFC preparation for $15 \mathrm{~min}$. with the acceptor culture, loopfuls of the mixture, and of dilutions of it, were plated on semi-solid medium containing streptomycin. There was no growth on control plates inoculated with the streptomycin-sensitive HFC preparation; on control plates inoculated with the acceptor strain there was growth only on the surface. On the plates inoculated with the mixture there appeared, in addition to surface growth, numerous microcolonies beneath the surface, up to $3 \mathrm{~mm}$. below it. We suppose that these colonies developed from streptomycinresistant non-motile acceptor bacteria carried into the depths of the medium by their motile streptomycin-sensitive partners. Many deep colonies were obtained from inocula of a 1/100 dilution, and a few from a $10^{-4}$ dilution of test mixtures in which the acceptor strain was either non-colicinogenic or was colicinogenic carrying colI. This confirmed our microscopical observation that bacteria of HFC preparations paired as readily with colicinogenic as with non-colicinogenic acceptor bacteria. When a pure culture of a motile, streptomycin-sensitive strain carrying colI was mixed with a culture of a non-motile, streptomycin-resistant acceptor, some deep colonies were produced from an inoculum of the undiluted mixture; but none from a 1/100 dilution. We infer that a broth culture of a strain carrying colI contains some bacteria able to adhere to acceptor bacteria, but far fewer than are present in an overnight mixed culture or HFC preparation; and too few to detect by microscopy. Presumably these few bacteria able to pair correspond to the about 1/5000 
'competent donors' able to transmit the colI factor in such cultures (Ozeki et al. 1962). No deep colonies were obtained from control mixtures of non-colicinogenic motile streptomycin-sensitive and non-colicinogenic non-motile streptomycinresistant strains.

Antigenic character of HFC preparations. The adherence of bacteria in HFC preparations to other bacteria presumably results from some alteration of their surface, which might cause some new antigenic specificity. A rabbit given two courses of inocula of an HFC preparation yielded sera with equal titres (1/6400) on O-agglutinable suspensions made from the HFC preparation and from the non-colicinogenic strain. After two absorptions with non-colicinogenic bacteria the sera no longer agglutinated either the $\mathrm{HFC}$ or the non-colicinogenic $\mathrm{O}$ suspension, even when tested undiluted. Thus in a very limited experiment we did not detect any new antigen in Salmonella typhimurium newly made colicinogenic for colI.

\section{Treatment of HFC preparations by high-speed blendor; kinetics of transfer of coll by HFC preparations}

Treatment of an HFC preparation in a high-speed blendor broke up all the clumps originally present; such treatment does not kill Salmonella typhimurium, but makes the organisms temporarily non-motile by breaking off their flagella (Stocker \& Campbell, 1959). Such deflagellated HFC preparations and untreated controls transmitted colI to about the same proportion of the bacteria of a motile acceptor strain in $1 \mathrm{hr}$. But when the acceptor strain was non-motile the deflagellated HFC preparation transmitted rather less well than did the untreated control. This presumably indicates that the collisions of donor and acceptor bacteria, which would be less frequent when both are non-motile, are rate-limiting under these conditions.

Although a mixture of an HFC preparation and an acceptor culture after $60 \mathrm{~min}$. incubation contained many clumps, the proportion of acceptor bacteria (selected by plating on streptomycin agar) which produced colicinogenic colonies was about the same when the mixture was de-clumped by blendor treatment before plating as when this step was omitted, probably because the spreading of the bacteria over the surface of the agar breaks up clumps about as well as does blendor treatment.

To study the time needed for transmission of colI, an HFC preparation and a streptomycin-resistant acceptor culture were mixed and incubated for 2 min. to allow pairing. The mixture was then gently diluted $1 / 100$ or $1 / 1000$ in broth, to preserve pairs already formed but to prevent further pairing; the dilute mixture was then incubated. At intervals samples were treated in the high-speed blendor to break up clumps and pairs, and either plated at once or after further incubation. It appeared (Table $4 a, b)$ : (i) that incubation of the undiluted mixture for $2 \mathrm{~min}$. sufficed for $10 \%$ of the acceptor population to pair with donors as against $30 \%$ in a control mixture incubated without dilution for $22 \mathrm{~min}$.; (ii) that in a small minority of pairs transfer of coll was completed (i.e. had reached a stage where separation of the partners did not affect the outcome) within 2 or $2 \frac{1}{2} \mathrm{~min}$. of mixing the cultures; (iii) that in most pairs transfer was completed by about $20 \mathrm{~min}$. after pair formation. 
Table 4. Kinetics of transfer of coll as determined by blendor treatment

(a) An HFC preparation, components adeC-7 proA-46 fla-(colI) and cysD-36 (colE2), and an acceptor culture, ath $A-4$ phe-401 str-r, were mixed in equal parts, and after 2 min. the mixture was diluted $1 / 100$ in broth. At intervals samples were treatedin the blendor and then plated on streptomycin agar. One sample of the mixture was incubated undiluted for $22 \mathrm{~min}$, then treated in blendor and plated.

\begin{tabular}{lccc} 
Sample & $\begin{array}{c}\text { Time of } \\
\begin{array}{c}\text { blendor } \\
\text { treatment, } \\
\text { (min.) }\end{array}\end{array}$ & \multicolumn{2}{c}{$\begin{array}{c}\text { Acceptor colonies } \\
\text { colicinogenic }\end{array}$} \\
\cline { 3 - 4 } Diluted & $2 \frac{1}{2}$ & $0 \cdot 8$ & Number \\
& $\mathbf{5}$ & $1 \cdot 8$ & $\mathbf{2} / 252$ \\
& 10 & $4 \cdot 1$ & $\mathbf{3} / \mathbf{7 7 8}$ \\
& 20 & $7 \cdot 6$ & $7 / 92$ \\
Undiluted & 35 & 10 & $9 / 90$ \\
& 22 & 31 & $27 / 86$
\end{tabular}

(b) An HFC preparation (components as in (a) above) was treated in the blendor, then mixed with an acceptor culture as above. 2 min. later the mixture was diluted 1/1000 in broth and at intervals samples were treated in the blendor. All samples were incubated until 75 min. from time of original mixing, then plated on streptomycin agar.

\begin{tabular}{|c|c|c|}
\hline \multirow{2}{*}{$\begin{array}{l}\text { Time of } \\
\text { blendor } \\
\text { treatment } \\
\text { (min.) after }\end{array}$} & \multicolumn{2}{|c|}{$\begin{array}{l}\text { Acceptor colonies } \\
\text { colicinogenic }\end{array}$} \\
\hline & $\%$ & number \\
\hline $\mathbf{3}$ & $2 \cdot 7$ & $7 / 259$ \\
\hline 10 & $5 \cdot 6$ & $19 / 338$ \\
\hline 25 & $7 \cdot 6$ & $31 / 408$ \\
\hline 45 & $8 \cdot 9$ & $23 / 258$ \\
\hline 75 & $10 \cdot 1$ & $36 / 348$ \\
\hline
\end{tabular}

\section{Factors affecting the transfer of colI from an HFC preparation to an acceptor culture}

Acceptor culture. When a suitable HFC preparation was incubated with an acceptor culture, the proportion of acceptor bacteria which acquired coll in $60 \mathrm{~min}$. was about the same, regardless of whether the acceptor suspension was from an overnight unshaken broth culture, an overnight shaken broth culture, or an overnight unshaken culture diluted tenfold in broth and incubated for $2 \mathrm{hr}$. (the more concentrated cultures being diluted, so that all three acceptor suspensions had about the same total count). Thus the physiological state of the acceptor bacteria appears to be unimportant. The proportion of bacteria of a non-motile acceptor strain which acquired coll during $1 \mathrm{hr}$. contact with an HFC preparation (of motile bacteria) was about the same as when the acceptor strain was motile.

Aeration. In an experiment in which the mixture of the HFC preparation and the acceptor bacteria was incubated on the shaker for $1 \mathrm{hr}$., the proportion of acceptor bacteria which acquired colicinogeny was $50 \%$, while in an unshaken control it was $32 \%$.

Medium. In the experiments described above the transfer of colI to acceptor bacteria took place in broth, the $\mathrm{HFC}$ preparation and acceptor culture being mixed 
without alteration of the medium. Fisher $(1957 a, b)$ observed that recombination in Escherichia coli $\mathrm{K} 12$ was influenced by the medium, being greatly decreased in liquid minimal medium lacking an energy source, and restored to normal by the addition of glucose and aspartate. We therefore looked for such effects on the transmission of colI. HFC and acceptor cultures were centrifuged and washed twice in minimal salts solution. Resuspension in fresh broth resulted in as good or nearly as good transfer of colI to acceptor bacteria as in control mixtures of the original broth cultures (e.g. $13 \%$ in 30 min., as compared with $18 \%$ in a control). Rather variable results were obtained when the washed HFC and acceptor cultures were resuspended and mixed in a minimal salts medium without a carbon source (minimal medium of Ozeki et al. 1962, but with glucose and citrate omitted). In one experiment (HFC strains $c y s D-36$ (colI) and $c y s D-36 \mathrm{col}^{-}$and acceptor strain adeC-7 proA-46 flastr-r) only about $0.4 \%$ of the acceptor bacteria acquired coll in $30 \mathrm{~min}$. (compared with $13 \%$ when resuspended in broth), and the addition of glucose $(0 \cdot 2 \%, w / v)$ to the final mixture only increased the degree of transfer to $1 \%$. In this experiment the addition of: aspartate $(0.02 \%, w / v)$; or of cystine $(0.002 \%, w / v)$, required by the donor strain; or of both adenine and proline (each $0.002 \%, \mathrm{w} / \mathrm{v}$ ), required by the acceptor strain, increased the rate of transfer to $9.5,10$ and $3 \%$, respectively.

However, in other experiments involving HFC and acceptor cultures twice washed and resuspended in minimal medium lacking citrate and glucose, there was good transfer (e.g. $44 \%$ in $30 \mathrm{~min}$.) when glucose was added to the mixture, and some transfer (e.g. $5 \%$ in 30 min.) in its absence. Previous starvation of one or both components by aeration for $1 \mathrm{hr}$. in minimal salts solution did not affect the transfer which occurred at a low rate in the absence of glucose but did depress the transfer in the presence of glucose. These results, however, were not consistent enough to permit conclusions to be drawn as to whether starvation of the donor or the acceptor, or of both, caused inhibition of the transfer of coll.

\section{Effect of metabolic inhibitors and of acriflavine}

Various metabolic poisons and acriflavine (which interferes with multiplication of the F agent in Escherichia coli; Hirota, 1960) were tested for ability to prevent the transmission of coll from HFC preparations to acceptor cultures (Table 5). The agents were added to HFC and acceptor cultures, separately, to allow them to establish their effect on bacterial metabolism before transmission could begin; after 11 min. equal parts of the treated HFC and acceptor cultures were mixed and the mixture incubated for $\mathbf{3 0}$ min.; samples were then treated in the blendor and plated. In a separate experiment an overnight broth culture was diluted 1/10 into tubes of broth containing the various agents. Turbidity measurements were made at intervals for $3 \mathrm{hr}$. to test the growth-inhibitory effect of the agents under these conditions, in which growth of the control culture is fairly rapid; the turbidity of the control culture increased $\times 13$ in this period. Streptomycin at $1 \mathrm{mg} . / \mathrm{ml}$. decreased coll transfer only slightly, to $64 \%$ of the control value in a mixture without streptomycin, even though this concentration was rapidly bacteriostatic and less rapidly bactericidal (20\% survival of acceptor bacteria after $1 \mathrm{hr}$. exposure). Dinitrophenol (M/500) and potassium cyanide (M/250) greatly inhibited growth but had little effect on the transfer of coll. Chloramphenicol (10 and $25 \mu \mathrm{g} . / \mathrm{ml}$.) though bacteriostatic only decreased transfer by about half. Sodium azide (M/100) 
did not inhibit growth during the first hour (although thereafter it did inhibit growth to some extent) but it did decrease transfer of colI to about $5 \%$ of the control value. Acriflavine ( $8 \mu \mathrm{g} . / \mathrm{ml}$.) had no effect on growth and little on transfer of colI acriflavine (40 and $80 \mu \mathrm{g} . / \mathrm{ml}$.) were growth-inhibitory and decreased transfer to 16 and $2 \%$ of the control value. However acriflavine $(40 \mu \mathrm{g} . / \mathrm{ml}$. $)$ was bactericidal and killed bacteria carrying coll more rapidly than non-colicinogenic ones (e.g. survivals of 7 and $53 \%$, respectively, after exposure to acriflavine $40 \mu \mathrm{g} . / \mathrm{ml}$. in broth for $2 \mathrm{hr}$.), so that some of its apparent effect in diminishing the rate of transfer of coll may have been spurious. Exposure of the HFC preparation alone to acriflavine $(40 \mu \mathrm{g} . / \mathrm{ml}$.$) , chloramphenicol (10 \mu \mathrm{g} . / \mathrm{ml}$.) or azide (M/200) for 15 or $30 \mathrm{~min}$., before washing and mixing with the acceptor culture, did not diminish the extent of transfer of colI as compared with an untreated control. Conversely, addition of the same concentration of acriflavine or chloramphenicol at the time of mixing the HFC preparation with the acceptor culture decreased the transfer of colI to about the same extent as when the separate HFC and acceptor cultures were pre-treated for $11 \mathrm{~min}$., but the inhibition of the transfer of colI by azide was less when pre-treatment was omitted.

\section{Table 5. Effect of metabolic inhibitors on transmission of coll by HFC preparations}

Components of HFC preparations adeC-7 pro A-46 fla- (colI) and $c y s D-36$ (colE2). Proportion of bacteria producing colicine I $21 \%$ (expt. 1) and $27 \%$ (expt. 2) after primary incubation, and $89 \%$ and $80 \%$, respectively, after secondary incubation. Inhibitors were added separately to HFC and acceptor cultures, which were mixed $11 \mathrm{~min}$. later. After 30 min. incubation the mixtures were diluted, treated in blendor, and plated on streptomycin agar, and the proportion of acceptor (streptomycin-resistant) colonies producing colicine I was determined. Acceptor strain: adeC-7 proA-46 fla- str-r (Expt. 1); athA-4 phe-401 str-r (Expt. 2).

Inhibitor

None

Potassium cyanide

Sodium azide

Dinitrophenol

Chloramphenicol

Streptomycin

Acriflavine
Concentration

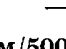

$\mathbf{M} / \mathbf{2 5 0}$

$\mathbf{M} / 200$

M/100

M/500

$10 \mu \mathrm{g} . / \mathrm{ml}$.

$25 \mu \mathrm{g} . / \mathrm{ml}$.

$1 \mathrm{mg} . / \mathrm{ml}$.

$80 \mu \mathrm{g} . / \mathrm{ml}$.

$40 \mu \mathrm{g} . / \mathrm{ml}$.

$8 \mu \mathrm{g} . / \mathrm{ml}$.

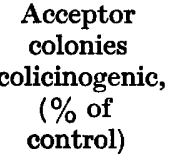

$100 * \dagger$

79*

$45 \dagger$

18*

$5 \cdot 4 \dagger$

$100 \dagger$

36*

$54 \dagger$

64.

$2 \dagger$

$19^{*}$

40*

* Expt. 1. $47 \%$ of acceptor colonies colicinogenic in control, without inhibitor.

$\dagger$ Expt. 2. $37 \%$ of acceptor colonies colicinogenic in control, without inhibitor. 


\section{Proportion of competent donors in HFC preparations}

In broth cultures of stock LT2 (colI) sublines only one bacterium in about 8000 is a competent donor able to transmit colI to an acceptor bacterium (Ozeki et al. 1962). To test the hypothesis that all or many bacteria which have just acquired colI can transmit, we determined the proportion of competent donors in HFC preparations in which a large fraction of the bacteria had acquired colI during the final $2 \mathrm{hr}$. of secondary incubation. The method used was like that used to determine the proportion of competent donors in broth cultures of established LT2 (coll) stocks (Ozeki et al. 1962). Graded numbers of bacteria from an HFC preparation of a streptomycin-sensitive strain were added to tubes containing $1 \mathrm{ml}$. of broth culture of a streptomycin-resistant acceptor strain $\left(7 \times 10^{8}\right.$ bacteria). After 20 or $30 \mathrm{~min}$. streptomycin (to $1 \mathrm{mg} . / \mathrm{ml}$.) was added, to prevent any growth of the streptomycin-sensitive donor bacteria, and the tubes after dilution $2 \frac{1}{2} \mathrm{hr}$. later with $4 \mathrm{ml}$. streptomycin broth were incubated for $18 \mathrm{hr}$. The tubes were then diluted in streptomycin broth and incubated for a further $24 \mathrm{hr}$., and thereafter tested for the presence of streptomycin-resistant (acceptor) bacteria producing colicine I. As in the experiments on stock colicinogenic strains (Ozeki et al. 1962) either 25-50\%, or apparently none, of the acceptor bacteria were colicinogenic when the incubated tubes of streptomycin broth were plated. In one experiment all tubes which received an average of $\mathbf{2 \cdot 3}$ colony-forming units or more from the HFC preparation contained colicinogenic acceptor bacteria. Of five tubes receiving an average of 0.7 colonyforming units only one tube contained colI str-r bacteria. Therefore an average of $2 \cdot 3$ colony-forming units sufficed to initiate the spread of colI. In this experiment the HFC preparation was not treated in the blendor to break up clumps before dilution, and the concentration of colony-forming units was determined by seeding drops on nutrient agar (Miles \& Misra, 1938) without spreading. It is therefore possible that an inoculum of $\mathbf{2 \cdot 3}$ colony-forming units represented a larger number of viable bacteria. In another experiment, in which the strains used were those shown in Table 6, the HFC preparation was treated in the blendor immediately before it was diluted. Inocula of the dilute HFC preparation were added to two series of tubes containing acceptor bacteria, so that the average number of bacteria inoculated per tube was 0.33 in one series and 0.83 in the second series, as determined by a subsidiary experiment in which $4 / 14$ and 8/14 tubes of broth which received the same inocula gave bacterial growth. After the addition of streptomycin broth and incubation as in the preceding experiment 4/42(0.09) tubes of the first series and $5 / 18(0 \cdot 28)$ tubes of the second series yielded colicinogenic acceptor bacteria. The average number of competent donor bacteria inoculated per tube calculated from these proportions is $\mathbf{0 . 0 9}$ for the first series and $\mathbf{0 . 3 3}$ for the second series. That is, about one bacterium in three $(0 \cdot 09 / 0 \cdot 33$ and $0 \cdot 33 / 0 \cdot 83)$ was a competent donor. In this experiment the proportion of colicinogenic bacteria in the donor preparation increased from 21 to $83 \%$ during the secondary incubation, so that about $62 \%$ of the bacteria of the HFC preparation had acquired coll during the final $2 \mathrm{hr}$. If only these newly-infected bacteria transmitted, then rather more than half of them must have been competent donors. 


\section{Duration of high infectivity after acquisition of coll}

When an HFC preparation was incubated without addition of fresh broth for $18 \mathrm{hr}$., or was stored at about $4^{\circ}$ for $18 \mathrm{hr}$., its transmitting ability decreased somewhat (22 and $16 \%$, respectively, of acceptor bacteria acquiring colI in $60 \mathrm{~min}$. contact, as compared with $\mathbf{5 7} \%$ for the fresh HFC preparation). But when the HFC preparation was diluted with broth even as little as $1 / 5$ and then incubated for $18 \mathrm{hr}$., the HFC property was lost ( $<1 \%$ acceptor bacteria made colicinogenic in $1 \mathrm{hr}$.). Broth cultures comprising all or most of the 1-2 $\times 10^{8}$ progeny of the 27th generation of single bacteria newly infected with colI were obtained, either (i) by inoculating broth with small acceptor colonies, later shown to have grown from acceptor bacteria which had acquired colI from an HFC preparation; or (ii) by incubating until just turbid $1 \mathrm{ml}$. samples of broths, each seeded with an inoculum containing an average of $<1$ bacterium from an HFC preparation. None of 23 cultures so obtained transmitted at high frequency. Thus under conditions which permitted rapid growth the high infectivity of bacteria newly infected with coll was not shown by their about 27 th generation progeny.

Table 6. Duration of high infectivity of an HFC preparation allowed to grow

An HFC preparation (components adeC-7 proA-46 fla- (colI) and cysD-36 (col E2)) was diluted to about $10^{4}$ bacteria $/ \mathrm{ml}$. and further diluted during incubation so as to keep the concentration between $10^{4}$ and $10^{5}$ bacteria $/ \mathrm{ml}$. At intervals $0.5 \mathrm{ml}$. inocula of graded numbers of bacteria were added to tubes containing $1 \mathrm{ml}$. of broth culture of a streptomycin-resistant acceptor culture (ath A-4 phe-401 str-r). Streptomycin to $1 \mathrm{mg}$. $/ \mathrm{ml}$. was added after 20 min and $5 \mathrm{ml}$. streptomycin broth at $2 \mathrm{hr}$. Tubes were tested for presence of streptomycin-resistant colicinogenic bacteria after overnight incubation; negative tubes were re-tested after further dilution and incubation in streptomycin broth.

Time of incubation of diluted HFC preparation (min.)

$\mathbf{0}$ 95 255

No. of*
generations
since
dilution

$\mathbf{0}$ $\mathbf{3}$ $7 \cdot 5$
Samples tested for ability to transmit

$\overbrace{\begin{array}{c}\text { Mean no. } \\ \text { bacteria/ } \\ \text { sample }\end{array}}^{\begin{array}{c}\text { Proportion } \\ \text { of tubes } \\ \text { positive }\end{array}}$

$\left\{\begin{array}{cc}670 & 5 / 5 \\ 67 & 5 / 5 \\ 6 \cdot 7 & 4 / 5\end{array}\right\}$

$\left\{\begin{array}{cc}460 & 5 / 5 \\ 46 & 5 / 5 \\ 4 \cdot 6 & 2 / 5\end{array}\right\}$

$\left\{\begin{array}{cc}4800 & 2 / 5 \\ 960 & 0 / 5 \\ 96 & 0 / 5 \\ 9 \cdot 6 & 0 / 5\end{array}\right\}$

Calculated $\dagger$ proportion of competent donors

$\frac{1 \cdot 6}{6 \cdot 7}=0.24$

$\frac{0.5}{4.6}=0.11$

$\frac{0.5}{4,800}=0.0001$

* Calculated from multiplication, as determined by viable counts.

$\uparrow$ Calculated from sample size giving some but not all positive tubes; mean no. of competent donors/sample calculated by Poissonian method from proportion of negative tubes, then divided by mean no. of bacteria/sample.

The number of generations for which high infectivity persists under conditions of rapid bacterial growth was determined as follows (Table 6). An HFC preparation after treatment in a high-speed mixer was diluted to about $10^{4}$ bacteria $/ \mathrm{ml}$., so that the frequency of collisions would be negligible, and incubated at $\mathbf{3 7} 7^{\circ}$. This culture was further diluted at intervals, so as to keep the concentration between $10^{4}$ and 
$10^{5}$ bacteria/ml. At various times graded inocula from the dilute culture were tested for ability to initiate the epidemic spread of the colI factor in a streptomycinresistant acceptor culture. At time zero about one bacterium in four was a 'competent donor'. After 3 generations of growth the proportion of competent donors had decreased from one in four to one in nine; thus about half the third generation progeny of the originally competent donors were themselves competent donors. After $7 \frac{1}{2}$ generations of growth the proportion of competent donors was only $0.01 \%$, which is about the proportion found in a broth culture of a stock colicinogenic strain. Thus most of the eighth generation clones (about 200 bacteria) derived from the originally competent donors did not include even a single competent donor.

\section{DISCUSSION}

The experiments described above show that cultures grown from suitable mixtures of colicinogenic and non-colicinogenic bacteria, that is HFC preparations, transmitted coll to a high proportion of the bacteria of an acceptor culture during 20-60 min. of contact, and that inocula of as few as 1 to 3 bacteria of a streptomycin-sensitive HFC culture sufficed to initiate the spread of colI amongst a streptomycin-resistant population in the presence of streptomycin. These observations and others show that a high proportion (between 30 and $100 \%$ ) of bacteria which had recently acquired colI were competent donors of colI, and that this competence persisted amongst their progeny for several generations (probably between 3 and 7 ) and then was abruptly lost. The only sort of culture able to transmit colI at high frequency will thus be one containing a high proportion of bacteria which have acquired coll within the last few generations, that is ones in which the 'epidemic' spread of colI is reaching or has just reached its peak.

The conditions required for the production of an HFC culture can then be understood in terms of the epidemiology of a contagious condition affecting the bacteria, and of the known changes in population density during incubation of broth cultures, unaerated or aerated. Presumably the spread of colI occurs to a significant extent only when the population density is high enough to permit frequent contacts of infective and susceptible bacteria. Transmission of colI by the small proportion of effective donors which occur in an established colicinogenic culture will then occur, and the newly infected recipient cells will become infectious and initiate epidemic spread amongst the susceptible population. On this hypothesis the function of the colicinogenic component of the mixed inoculum is to provide sufficient competent donors to initiate the spread of colI amongst the non-colicinogenic bacteria when the bacterial population density becomes high enough for collisions to be frequent. With a suitable constant ratio of colicinogenic to non-colicinogenic bacteria, variation in the absolute number of bacteria inoculated would be expected to have little effect on the progress of the colI epidemic, since in any event transmission would only occur at a significant rate after the bacterial population density reached a high level. Similarly, the number of colicinogenic bacteria inoculated together with a fixed number of non-colicinogenic bacteria, provided that the former were in a minority, would affect only the time of onset of the epidemic. We found that colicinogenic:non-colicinogenic inoculum ratios between $1: 200$ and 1:1 gave satisfactory HFC preparations. Presumably the failure to obtain HFC preparations 
when the proportion of colicinogenic bacteria in the inoculum was still smaller resulted from a delay in the onset of the epidemic. When the inoculum contained more colicinogenic bacteria than non-colicinogenic ones, HFC preparations were not obtained, and the proportion of the non-colicinogenic component which acquired colI was very small; in these circumstances the majority of collisions of infective donors would be with bacteria already carrying coll, which would not themselves become effective donors (see Ozeki et al. 1962). In epidemiological terms the high proportion of immunes in the population will decrease the population density of susceptibles and thus impede the progress of the epidemic.

Mixed inocula of non-motile colicinogenic and motile non-colicinogenic bacteria gave satisfactory HFC preparations; but when the colicinogenic component was motile and the non-colicinogenic was non-motile, the usual incubation procedure did not give HFC preparations (Table 3). In these two situations the frequency of collision of effective donors of the colicinogenic component with non-colicinogenic bacteria would be the same, but in all of many subsequent cycles of infection in the initially non-colicinogenic strain the average time before an effective donor collided with a susceptible bacterium would be increased when the non-colicinogenic strain was non-motile; this would account for the delayed spread of colI in this situation observed when secondary incubation was prolonged (Table 3).

The $2 \mathrm{hr}$. 'secondary incubation' of a $1 / 10$ dilution of an overnight mixed culture provides a period in which population density is high and metabolism active, so that conditions are favourable for infection of a large proportion of the remaining susceptibles (usually about $70 \%$ of the bacteria of the initially non-colicinogenic strain).

The high transmitting ability of broth cultures inoculated directly from old stock slopes of a Salmonella paratyphi B strain carrying coll we attribute to the instability of colicinogeny in respect of colI in this strain. The accumulation of non-colicinogenic bacteria during storage results in the presence of a high proportion of bacteria susceptible to the epidemic spread of coll when material from the stock slope is incubated in broth. The failure of the non-colicinogenic bacteria to become reinfected in the stock slope itself presumably results from conditions there being unsuitable for the transmission of colI, perhaps because of lack of available nutrients (compare the absence of spread of coll in mixtures of fully grown aerated donor and acceptor cultures; Ozeki et al. 1962).

Our experiments in which inocula of graded numbers of streptomycin-sensitive bacteria from HFC preparations were tested for their ability to transmit in the presence of streptomycin indicate that of bacteria infected by colI within the previous $2 \mathrm{hr}$. about one-half to one-third can transmit. These experiments presented various technical difficulties. In particular we could not prepare suspensions containing bacteria all of which had carried coll for a known short period. We suspect that in consequence the proportion of newly infected bacteria which are competent donors was underestimated, and may in fact well be 1 , or near it, rather than 0.5 or $\mathbf{0 . 3}$, as estimated. Our attempts to measure the duration of high infectivity when newly infected bacteria were allowed to multiply were made difficult by the same experimental limitations. However, the data obtained (Table 6) seem to establish that for several generations a high proportion (probably at least half and perhaps more) of the immediate progeny of a competent newly infected 
bacterium are themselves competent donors; and that this high infectivity persists for 3 to 7 generations. The observed decrease in the proportion of competent donors from about 1 in 4 at the time of dilution of an HFC preparation to 1 in 9 three generations after dilution, and to about $0.01 \%$ after $7 \frac{1}{2}$ generations, permits some inference about the mechanism of the loss of competence. These data indicate that of the 8 third-generation progeny of a competent donor, an average of at least 4 are competent; but that an average of fewer than 1 (about 0.08 ) of the 200 seventhto eighth-generation progeny are competent donors. If the possession of at least one stable but non-replicating particle synthesized only by newly infected bacteria (e.g. a non-chromosomal colI particle) sufficed to confer competence, then the clone produced by a newly infected bacterium, since it contains 4 competent donors and therefore 4 particles at the 8 bacterium stage, should contain at least 4 competent donors amongst the 200 descendants after 7 to 8 generations; but in fact the average number of competent donors at this stage was $0 \cdot 08$. Therefore the hypothesis stated above is excluded. It seems more likely that at least half, perhaps all, the descendants of a competent newly infected bacterium are competent donors, but that after 3 to 7 generations every one of the progeny changes over to the non-competent state characteristic of established colicinogenic cultures.

The clumps seen by microscopy in HFC preparations and the way in which nonmotile acceptor bacteria are carried through a semi-solid medium by motile bacteria of an HFC culture show that in cultures which contain a high proportion of competent donors many bacteria adhere to other bacteria with which they have collided. The kinetic experiments show that blendor treatment (which breaks up such clumps and pairs) given immediately after pair formation has been permitted, prevents the transfer of coll. Pair formation is very rapid (about one-third completed in 2 min., when one or both strains are motile) and transfer of colI in a very few pairs is completed (i.e. has reached a stage unaffected by blendor treatment) within $2 \frac{1}{2} \mathrm{~min}$.; but in most pairs the transfer is not completed until at least $20 \mathrm{~min}$. after pair formation. Perhaps a variable time is required for the formation of a conjugation canal linking the interior of the donor and recipient bacteria, or for the movement of a colI particle through such a canal. The adherence of newly infected bacteria suggests that their surface may be altered; but in a very limited experiment we did not detect any new antigenic specificity in such bacteria. Observations on clump formation and on the carriage of streptomycin-resistant non-motile acceptor bacteria by their HFC partners away from the site of inoculation indicate that competent donor bacteria pair with bacteria of established colicinogenic strains carrying coll about as readily as they do with non-colicinogenic bacteria, and the extent of clumping in HFC preparations suggests that newly infected bacteria also adhere to each other.

Our experiments on the effect of various factors on the extent of transfer of colI by HFC preparations show that the physiological state of the acceptor bacteria is unimportant; this suggests that their role in transfer is a passive one. Aeration by shaking did not interfere with transmission; this supports our conclusion (Ozeki et al. 1962) that the inhibitory effect of aeration by shaking on the transfer of colI during the prolonged incubation of mixtures of donor and intermediate strains in broth is a result not of a direct effect on pair formation or transfer, but an indirect one, through shortening the period of growth at a high bacterial concentration. The 
failure of aeration to inhibit such transfer in a defined medium may have been due to the longer generation time in such a medium, so that even in aerated cultures there is a longer period of growth at a high bacterial concentration to allow transfer of coll. Our experiments on the environmental requirements for transfer showed that bacteria which had been infected by coll during growth in broth could transfer coll to some extent when resuspended in a defined medium (unlike the transfer of the F agent in Escherichia coli; Cavalli, Lederberg \& Lederberg, 1953) but that there was only slight transfer when the defined medium contained no energy source. Furthermore, our ability to obtain HFC preparations by growth in defined medium shows that newly infected bacteria become competent donors of coll in a defined medium, as well as in broth. Our results with prior starvation of one or the other partner did not establish whether it is only the donor strain which requires an energy source (external or internal), as in the case of chromosomal transfer from $E$. coli $\mathrm{Hfr}$ to $F^{-}$(Fisher, 1957b).

Our experiments on the effect of metabolic inhibitors, etc., on the transfer of colI showed there was little correlation between their ability to inhibit growth and to prevent transfer. Thus cyanide, dinitrophenol and streptomycin at growth-inhibitory concentrations had little effect on transfer, whereas azide (M/100) almost entirely prevented transfer without slowing growth. The failure of cyanide and dinitrophenol to prevent transfer of colI in Salmonella typhimurium to any major extent contrasts with their ability, at lower concentrations, to prevent chromosomal recombination in Escherichia coli $\mathrm{K12}$ (Fisher, 1957a); but it is not clear whether this difference results from a species difference or from a difference in the susceptibility of chromosomal transfer or of episomal transfer (there being no data on the transmission of $\mathbf{F}$ in $E$. coli in the presence of inhibitors). Only growthinhibitory concentrations of acriflavine suppressed transfer and we have some data suggesting that it preferentially kills colicinogenic bacteria; thus we have no evidence for a specific effect on the multiplication of colI, comparable to the effect of acriflavine on the multiplication of the F factor in E. coli (Hirota, 1960). Streptomycin $1 \mathrm{mg} . / \mathrm{ml}$. only diminished transfer by $36 \%$; we observed that $S$. typhimurium remained motile for several hours in this concentration, which suggests that it leaves unaffected at least some energy-yielding mechanisms. In our experiments on the proportion of competent donors in stock colicinogenic strains (Ozeki et al. 1962) and in HFC preparations, the time available for transfer of coll by any competent donors in the inoculum of streptomycin-sensitive bacteria was therefore longer than the $\mathbf{2 0}$ or $\mathbf{3 0} \mathrm{min}$. before streptomycin was added. But since streptomycin $1 \mathrm{mg} . / \mathrm{ml}$. immediately arrests multiplication of strain LT2 the inferences drawn as to the number of competent donors are unaffected.

We have above assumed that broth cultures of stock colicinogenic strains carrying coll are heterogeneous, containing a very few competent donor bacteria able to pair and transmit coll, amidst a large majority unable to do so; and that of the newly infected bacteria present in HFC preparations a large fraction (e.g. 0.2-0.5) or perhaps all, can pair and transmit, the remainder, if any, being unable to do so. Our data however do not exclude the hypothesis that each of these bacterial populations is, on the contrary, homogeneous, every newly infected bacterium having the same probability of pairing and transmitting colI (under the conditions of our experiments, see above, between 0.3 and 1 ), the corresponding probability for 
every bacterium in a stock colicinogenic strain being only about $10^{-4}$. However, the rare competent donors in stock colicinogenic cultures behave, so far as we have tested, just like the frequent competent donors in HFC preparations; for instance, in usually transmitting, as well as colI, any other colicine factor they may possess (Ozeki et al. 1962; Smith, Ozeki \& Stocker, in preparation). For this reason we prefer the hypothesis of two possible phenotypes for bacteria carrying colI: the effective donors, having a probability of one, or near one, of mating and transmitting under suitable conditions; and other colicinogenic bacteria, with zero probability of doing so. Our uncertainty as to the homogeneity or heterogeneity of transmitting ability in our bacterial populations does not, we think, affect the validity of the rest of our argument.

From the rapid spread of colI during the growth of HFC cultures it is evident that in such cultures the colI factor is multiplying much more rapidly than the bacteria, and therefore that in newly infected bacteria colI multiplies autonomously. As the large majority of bacteria in stock colicinogenic strains do not transmit coll we have no evidence that in them colI is multiplying more rapidly than its bacterial host. These non-transmitting bacteria do not adhere to other bacteria, as shown by the absence of clumping and by the rarity of satellite colonies when a mixture of a streptomycin-resistant non-motile acceptor culture and a stock streptomycinsensitive motile colicinogenic culture is plated on semi-solid streptomycin medium. The failure of such bacteria to adhere to other bacteria with which they collide seems a sufficient explanation for their failure to transmit colI. We do not know why the colI factor in most newly infected bacteria manifests the F-like property of causing its bacterial host to conjugate, but does so in only a very small minority of bacteria of strains which have long harboured it. Perhaps the colI factor when first introduced multiplies freely in the cytoplasm but after some generations is nearly always present only as a single particle attached to the chromosome; either a 'dose-effect' or a 'position-effect' might then account for the usual failure of the attached colI factor to confer 'maleness' on its host. If this be so, coll in newly infected Salmonella typhimurium would resemble the non-integrated $\mathbf{F}$ factor in Escherichia coli $\mathrm{F}^{+}$strains; and the colI agent in established $S$. typhimurium colicinogenic strains would resemble the chromosomally attached $F$ agent in $E$. coli Hfr strains (though the integrated $F$ episome continues to confer the male phenotype on its Hfr host). However, there is as yet no evidence that coll (or any other colicine agent so far investigated) can be integrated into the chromosome of $S$. typhimurium. The failure to transmit which sets in between 3 and 7 generations after invasion by colI may result not from integration of the episome into the chromosome but from some other change causing loss of ability to conjugate. However preliminary experiments (Dubnau \& Stocker, unpublished) indicate that when an Hfr subline of $S$. typhimurium LT2 (Zinder, 1960) made colicinogenic for coll transfers the $i l e^{+}$gene to a non-colicinogenic ile- str-r LT2 acceptor strain most ile $e^{+}$str-r recombinants do not acquire colI; whereas in the recombination experiments of Ozeki \& Howarth (1961), in which HFC preparations were used as gene donors, the majority of recombinants did acquire coll. This argues against the autonomous multiplication of colI in the cytoplasm of bacteria of stock colicinogenic strains.

In Escherichia coli $\mathrm{F}^{-}$strains bacteria newly infected by the $\mathbf{F}$ agent or by colicine 
factors have not been shown to differ from stock $F^{+}$or colicinogenic strains. In Salmonella typhimurium, however, the immediate progeny of bacteria newly infected by the temperate phage PLT22 differ in two respects from bacteria of established lysogenic strains carrying prophage PLT22 : (1) They produce a high proportion of nonlysogenic descendants which after a delay revert to phage sensitivity (Luria, Fraser, Adams \& Burrous, 1958). (2) All or most of them manifest the phage-determined antigenic specificity called Factor 1 , attributable to the presence of a glucose- $\alpha-1,6-$ galactose-mannose side-chain in the polysaccharide of their $\mathbf{O}$ antigen (Stocker et al. 1960), whereas in established lysogenic strains the expression of Factor 1 is subject to form-variation (Kauffmann, 1940), so that many lysogenic bacteria are totally unaffected by Factor 1 serum (Stocker \& de Margerie, unpublished). We do not know why bacteria recently infected by coll or by prophage PLT22 should behave differently from bacteria of established colicinogenic or lysogenic strains. In each case the two states, that is of newly infected and of established strains, are distinct from the situation when the episome becomes more active, either spontaneously or after ultraviolet irradiation, and directs the synthesis of either colicine or infective phage, with the consequent death of the bacterial host. (See Ozeki, Stocker \& de Margerie (1959) for production of colicine, spontaneously or after induction, by individual bacteria in cultures of colicinogenic $S$. typhimurium LT2 sublines.)

\section{REFERENCES}

Cavalu, L. L., Lederberg, J. \& Lederberg, E. M. (1953). An infective factor controlling sex compatibility in Bacterium coli. J. gen. Microbiol. 8, 89.

Clowes, R. C. (1958). Investigation of the genetics of cysteineless mutants of Salmonella typhimurium by transduction. J. gen. Microbiol. 18, 154.

Demerec, M., Hartman, Z., Hartman, P. E., Yura, T., Gots, J. S., Ozeki, H. \& Glover, S. W. (1956). Genetic studies with bacteria. Publ. Carneg. Instn, no. 612.

Fisher, K. W. $(1957 a)$. The role of the Krebs cycle in conjugation in Escherichia coli K12. J. gen. Microbiol. 16, 120.

FishER, K. W. (1957b). The nature of the endergonic processes in conjugation in Escherichia coli K12. J. gen. Microbiol. 16, 136.

Hartman, P. E., Mudd, S., Hillier, J. \& Beutner, E. H. (1953). Light and electron microscopic studies of Escherichia coli-coliphage interactions. III. Persistence of mitochondria and reductase activity during infection of Escherichia coli B with T2 phage. J. Bact. 65, 706.

Hirota, Y. (1960). The effect of acridine dyes on mating type factors in Escherichia coli. Proc. nat. Acad. Sci., Wash. 42, 574.

InNo, T. (1958). Cistron test of motility genes in Salmonella. Rep. nat. Inst. Genet., Japan, 9, 96.

Kauffmann, F. (1940). Zur serologie des I-antigens in der Salmonella-gruppe. Acta path. microbiol. scand. 17, 135.

Luria, S. E., Fraser, D. K., Adams, J. N. \& Burrous, J. W. (1958). Lysogenisation, transduction and genetic recombination in bacteria. Cold Spring Harb. Symp. quant. Biol. 23, 71 .

Meynell, E. W. (1961). A phage, $\phi \chi$, which attacks motile bacteria. J. gen. Microbiol. 25, 253.

Mires, A. A. \& Misra, S. S. (1938). The estimation of the bactericidal power of the blood. J. Hyg., Camb. 38, 732.

Miyake, T. \& Demerec, M. (1960). Proline mutants of Salmonella typhimurium. Genetics, $45,755$.

ØrSkov, I. \& ØRSKov, F. (1960). An antigen termed $\mathrm{f}^{+}$occurring in $\mathrm{F}^{+} \boldsymbol{E}$. coli strains. Acta path. microbiol. scand. 48, 37. 
Ozekr, H. \& HowarTh, S. (1961). Colicine factors as fertility factors in bacteria: Salmonella typhimurium strain LT2. Nature, Lond. 190, 986.

Ozeki, H., Stocker, B. A. D. \& De Margerie, H. (1959). Production of colicine by single bacteria. Nature, Lond. 184, 337.

Ozeki, H., Stocker, B. A. D. \& Smrtr, S. M. (1962). Transmission of colicinogeny between strains of Salmonella typhimurium grown together. J. gen. Microbiol. 28, 671.

Quadirng, C. \& STocker, B. A. D. (1957). The occurrence of rare motile bacteria in some non-motile Salmonella strains. J. gen. Microbiol. 17, 424 .

Sertic, V. \& Boulgakov, N. A. (1936). Bactériophages spécifiques pour des variétés bactériennes flagellées. C.R. Soc. Biol., Paris, 123, 887.

Stocker, B. A. D. \& CAmprenl, J. C. (1959). The effect of non-lethal deflagellation on bacterial motility and observations on flagellar regeneration. J. gen. Microbiol. 20, 6ro.

Stocker, B. A. D., Staub, A. M., Tinelui, R. \& Kopacka, B. (1960). Étude immunochimique sur les Salmonella. VI. Étude de l'antigène 1 présent sur deux Salmonella des groupes $\mathrm{B}$ et $\mathrm{E}_{4}$. Ann. Inst. Pasteur, 98, 505.

Stocker, B. A. D., Zinder, N. D. \& Lederberg, J. (1953). Transduction of flagellar characters in Salmonella. J. gen. Microbiol. 9, 410.

Zinder, N. D. (1960). Sexuality and mating in Salmonella. Science, 131, 924. 